\section{Separation and Detection of Macrolide Antibiotics by HPLC Using Macrolide-imprinted Synthetic Polymers as Stationary Phases}

\author{
Martin Siemann $^{\dagger}$, Lars I. Andersson ${ }^{\dagger \dagger}$ \\ and KlaUs MosbaCH*
}

Pure and Applied Biochemistry, Chemical Center, University of Lund,

P.O. Box 124, S-221 00 Lund, Sweden

(Received for publication June 17, 1996)

Erythromycin and oleandomycin are macrolide antibiotics belonging to the erythromycin type and tylosin is one of the compounds of the structurally very related carbomycin family ${ }^{1}$ (Fig. 1). In particular erythromycin has, since its discovery in the early $1950 \mathrm{~s}$, been one of the very widely used antibiotics, useful in the treatment of a variety of infectious deseases ${ }^{2 \sim 4)}$. Several methods for the determination of these antiobitics in plasma and tissue, including microbial test systems ${ }^{5)}$, radioimmunoassay $^{6)}$ and liquid chromatography ${ }^{7,8)}$, have been described.

Molecular imprinting ${ }^{9}$ of organic compounds is increasingly recognised as a facile technique for the preparation of polymeric materials containing recognition sites of predetermined specificity. The resulting polymers retain a memory for the print molecule and can be used in the HPLC mode for separation of structurally similar substances, and (in case of single enantiomer imprinting) resolution of the enantiomers of the print species ${ }^{10}$. Polymerisation of monomers, which are selected for their ability to engage in non-covalent interactions with specific functionality of the print molecule, constitutes the key step. Subsequent removal of the print molecule exposes sites within the polymer matrix whose chemical and steric topography is defined by the print molecule present during its preparation. These memory sites enable the polymer to later rebind the print molecule, in many instances with binding affinities and specificities approaching those demonstrated by antigen-antibody systems ${ }^{11,14}$. This paper describes the molecular imprinting of some macrolide antibiotics and the use of the resultant polymers as stationary phases with predetermined selectivity in HPLC.

\section{Materials and Methods}

\section{Polymer Preparation}

Polymers were prepared according to a method described previously ${ }^{10)}$. Imprint molecule; $149 \mathrm{mg}(0.20$ mmol) of erythromycin A, $151 \mathrm{mg}(0.22 \mathrm{mmol})$ of oleandomycin, or $183 \mathrm{mg}(0.17 \mathrm{mmol})$ of tylosin, $3.96 \mathrm{~g}$

Fig. 1. Structures according to MERCK INDEX ${ }^{13)}$ of the macrolides antibiotics.

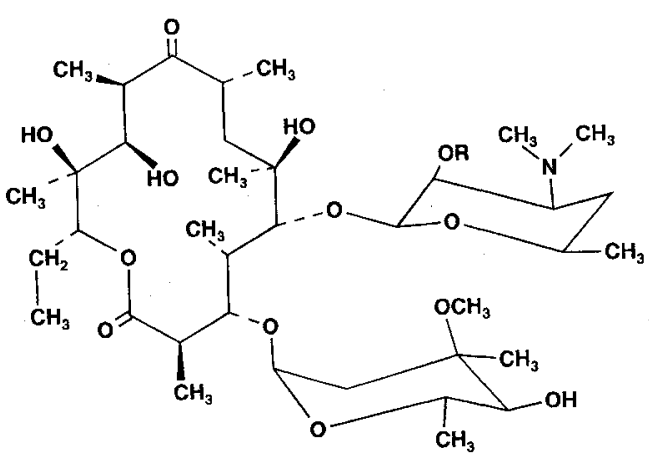

Erythromycin A $\mathrm{R}=\mathrm{H}$

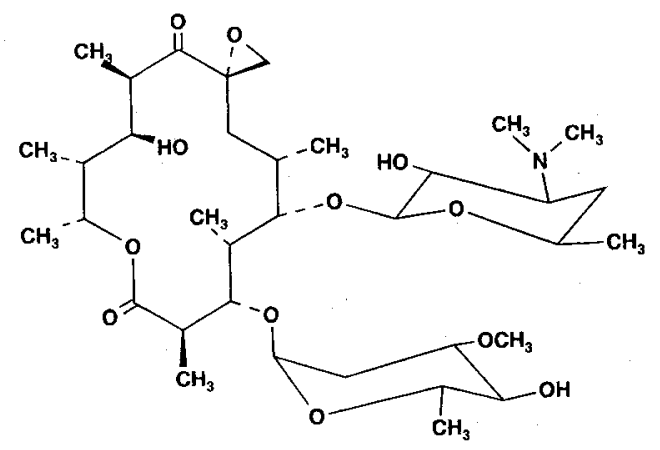

Oleandomycin

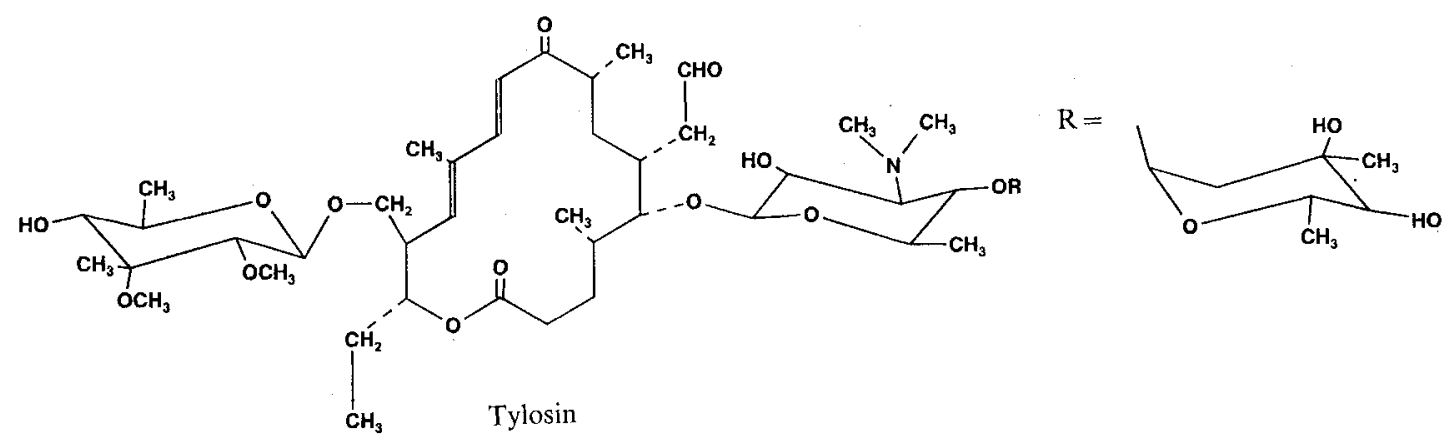

Present address: + Institute of Biochemical Engineering, University of Stuttgart, Allmandring 31, 70569 Stuttgart, Germany. $\dagger \dagger$ Astra Pain Control, Pharmaceutical and Analytical R \& D, Bioanalytical Chemistry, S-151 85 Södertälje, Sweden. 
of ethylene glycol dimethacrylate and $344 \mathrm{mg}$ of methacrylic acid were weighed into $20 \mathrm{ml}$ borosilicate test tubes and dissolved in $6 \mathrm{ml}$ of dried methylene chloride. A blank polymer was produced by omitting the print molecule. The mixtures were cooled on ice, sonicated and sparged with nitrogen for 5 minutes. Fifty mg of azobis(isobutyronitrile) (initiator) was added and the ampoules were placed under a UV-source $(366 \mathrm{~nm})$ for 16 hours at $4^{\circ} \mathrm{C}$. The bulk polymers were ground in a mechanical mortar (Retsch, Haan, Germany) and wet-sieved through a $25 \mu \mathrm{m}$ sieve (Retsch, Haan, Germany). Fines were removed by repeated sedimentation from acetonitrile and the polymer particles were finally dried under vacuum.

\section{HPLC Analysis}

Polymer particles were packed into stainless steel columns $(200 \times 4.6 \mathrm{~mm})$ as described in ${ }^{10)}$. In order to extract the imprint species, the columns were washed on line with methanol - acetic acid $(9: 1 ; \mathrm{v} / \mathrm{v} ; 1$ liter $)$ and then with the eluent. Twenty $\mu \mathrm{l}$ of $0.1 \mathrm{~mm}$ samples (dissolved in the eluent) were analysed isocratically at a flow rate of $1 \mathrm{ml} /$ minute. The eluents were: $0.5 \%$ acetic acid in chloroform (erythromycin polymer); $1 \%$ acetic acid in chloroform (oleandomycin polymer); $1 \%$ acetic acid in methylene chloride (tylosin polymer). Acetone was used as a void marker. Retention factors were calculated using $\mathrm{k}^{\prime}=\left(\mathrm{V}_{\mathrm{R}}-\mathrm{V}_{0}\right) / \mathrm{V}_{0}$, where $\mathrm{V}_{\mathrm{R}}$ is the retention volume of the compound and $\mathrm{V}_{0}$ is the void volume of the system. Separation factor is $\alpha=k_{1}^{\prime} / k_{2}^{\prime}$, where $k_{1}^{\prime}$ is the retention factor recorded for the print species and $\mathrm{k}_{2}^{\prime}$ is that for the second compound. Plate numbers $(\mathrm{N})$, which were around $5000 \mathrm{~m}^{-1}$ for all columns, was calculated using standard chromatographic theory ${ }^{12)}$.

\section{Results and Discussion}

Molecular imprinting of macrolide antibiotic structures were done in methacrylic acid-ethylene glycol dimethacrylate copolymers. The resultant molecularly imprinted polymers (MIPs) were packed into HPLCcolumns and their specific separation abilities were analysed, as has been described in previous studies of imprinted polymers ${ }^{10)}$. For each compound the retention factor, $\mathrm{k}^{\prime}$, is related to the relative binding affinity of the compound to the imprinted polymer. The extent of retention of a given compound on a column is, however, composed of two factors: the specific binding to the imprinted sites and non-specific retention due to interaction with the surface of the polymer. Non-specific interactions could be reduced to a minimum by addition of small amounts of acetic acid to the eluent ${ }^{10}$.

All polymers showed strongest affinity for the compound that was present during its preparation (Table 1). In all instances, other macrolides were less well recognised, i.e. other macrolides eluted earlier than the print species. On the column packed with oleandomycin MIP, the print molecule was completely separated from mixtures with erythromycin and tylosin, with separation factors $(\alpha)$ of 6.8 and 5.7, respectively. The same is true for erythromycin and tylosin MIPs. On the column packed with erythromycin MIP, erythromycin is more strongly retained than oleandomycin and tylosin $(\alpha=5.0$ and 2.4 , respectively). On the tylosin column, a much higher retention factor was recorded for tylosin than for oleandomycin and erythromycin; in this instance $\alpha=$ 8.2 and 2.9 were obtained. A non-imprinted reference polymer, made under otherwise identical conditions but by omitting the print molecule, was not able to retain any of the compounds tested. With the same mobile phase as used for the imprinted columns, all compounds eluted with the void $\left(\mathrm{k}^{\prime}=0.0\right)$. In all instances, the increased retention of the imprint species relative to other compounds is high, indicating a high specificity of each MIP. In this context, unrelated compounds, such as cyclosporin and rifampicin, eluted with the void $\left(\mathrm{k}^{\prime}=0.0\right)$ or was slightly retained $\left(\mathrm{k}^{\prime}=1.0\right)$ on the oleandomycin polymer (Table 1), which further demonstrate the specificity of the imprints. The slight retention of rifampicin may be explained by some structural similarities to the ansa chain of rifampicin to the macrolide ring of the imprint species. The very high separations obtained make these polymers useful in

Table 1. Chromatographic data obtained by HPLC analysis of imprinted polymers.

\begin{tabular}{|c|c|c|c|c|c|c|}
\hline \multirow{3}{*}{ Test compound } & \multicolumn{6}{|c|}{ Imprint molecule } \\
\hline & \multicolumn{2}{|c|}{ Oleandomycin } & \multicolumn{2}{|c|}{ Erythromycin } & \multicolumn{2}{|c|}{ Tylosin } \\
\hline & $\mathbf{k}^{\prime}$ & $\alpha$ & $\mathrm{k}^{\prime}$ & $\alpha$ & $\mathrm{k}^{\prime}$ & $\alpha$ \\
\hline Oleandomycin & 6.8 & 1.0 & 1.4 & 5.0 & 1.3 & 8.0 \\
\hline Erythromycin & 1.0 & 6.8 & 7.0 & 1.0 & 3.7 & 2.7 \\
\hline Tylosin & 1.2 & 5.7 & 2.9 & 2.4 & 10.7 & 1.0 \\
\hline Erythromycin estolate & 1.3 & 5.2 & 0.7 & 10.0 & 0.3 & 35.7 \\
\hline Erythr. ethyl-succinate & 0.9 & 7.6 & 1.7 & 4.1 & 0.1 & 107.0 \\
\hline Erythromycin stearate & 2.0 & 3.4 & 0.7 & 10.0 & 1.0 & 10.7 \\
\hline Spiramycin & 1.2 & 5.7 & 2.7 & 2.6 & 9.6 & 1.1 \\
\hline Rifampicin & 1.0 & 6.8 & n.d. & n.d. & n.d. & n.d. \\
\hline Cyclosporin & 0.0 & $\infty$ & 0.0 & $\infty$ & 0.0 & $\infty$ \\
\hline
\end{tabular}

Retention factor, $\mathrm{k}^{\prime}$, and separation factor $\alpha$, were calculated as described in Materials and Methods. n.d.: not determined. 
Fig. 2. Separation of a mixture of each $1 \mathrm{mg} / \mathrm{ml}$ of cyclosporin, erythromycin and oleandomycin, and $0.1 \mathrm{mg} / \mathrm{ml}$ of tylosin on the polymer with the imprints against tylosin.

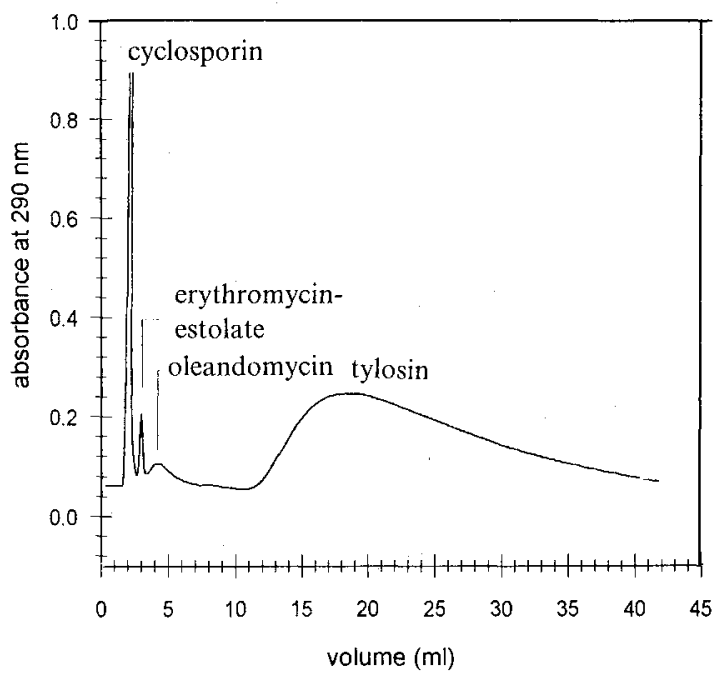

The eluent was methylene chloride containing 1\% acetic acid at a flow rate of $1 \mathrm{ml} / \mathrm{minute}$. Detection was at $290 \mathrm{~nm}$. Note the strong adsorption of tylosin at this wavelength.

chromatographic separations of the imprint species from a mixture of antibiotics and related compounds (Fig. 2). The data presented here demonstrate the ability of molecular imprinting to provide an easy access to stationary phases with high specificity of the desired macrolide structure.

At least in one instance, the polymer was able to detect subtle structural differences. On the erythromycin column, 2 -esters of erythromycin eluted much earlier than erythromycin itself did. The parent compound was separated from its ethyl succinate, stearate and estolate ester derivatives with separation factors of 7.6, 5.2 and 3.4, respectively (Table 1). In contrast, the bulky molecules tylosin and spiramycin gave similar retention values on the tylosin column, with spiramycin eluting only slightly prior to tylosin (Table 1). A possible explanation is their identical disaccharide unit combined with the many structural similarities in the macrocyclic ring.

In conclusion, we have demonstrated the molecular imprinting of macrolide antibiotics, a structurally complex class of compounds having a large ring structure containing many chemical functionalities. The resultant polymer demonstrated efficient recognition of their respective imprinted macrolides and may eventually be used in a chromatographic mode for analytic purposes or isolation of these antibiotics from fermentation broths.

\section{References}

1) Keller-Schierlein, W.: Chemie der Makrolith-Antibiotika. Fortschr. Chem. Org. Naturst. 30: 313 460, 1973

2) Washington, J. A. \& W. R. Wilson: Erythromycin: A microbial and clinical perspective after 30 years of clinical use (part 1). Mayo Clin. Proc. 60: 189 203, 1985

3) Washington, J. A. \& W. R. Wilson: Erythromycin: A microbial and clinical perspective after 30 years of clinical use (part 2). Mayo Clin. Proc. 60: 271 278, 1985

4) Westphal, J. F.; D. Vetter \& J. M. Brogard: Hepathic side-effects of antibiotics. J. Antimicrob. Chemother. 33: $387 \sim 401,1994$

5) Huck, T. A.; N. Porter \& M. E. Bushel: Positive selection of antibiotic-producing soil isolates. J. Gen. Microbiol. 137: $2321 \sim 2329,1991$

6) Tanaka, Y.; K. Kimura, Y. Komagata, K. Tsuzuki, H. TOMADA \& S. OMURA: Radioimmunoassay for erythromycin derivatives. J. Antibiotics 41: $258 \sim 260,1988$

7) Khan, K.; J. Paesen, E. Roets \& J. Hoogmartens: Analysis of erythromycin A and its metabolites in biological samples by liquid chromatography with post-column ion-pair extraction. L. Liquid Chrom. 17: 4195 4212, 1994

8) JANECEK, M; M. A. Quilliam, M. R. Bailey \& D. H. NORTH: Determination of erythromycin $A$ by liquid chromatography and electrochemical detection, with application to salmon tissue. J. Chromat. Biomed. Appl. 619: $63 \sim 69,1993$

9) MosBaCH, K.: Molecular imprinting. Trends Biochem. Sci. 19: $9 \sim 14,1994$

10) ANDERSSON, L. I. \& K. MosBaCh: Enantiomeric resolution on molecularly imprinted polymers prepared with only non-covalent and non-ionic interactions. J. Chromatogr. 516: $313 \sim 322,1990$

11) Vlatakis, G.; L. I. Andersson, R. Müller \& K. MosBaCH: Drug assay using antibody mimics made by molecular imprinting. Nature 361: 645 647, 1993

12) Unger, K. K.: Packings and stationary phases in chromatographic techniques. In Chromatographic Science Series, 47: Marcel Dekker, New York, Basel, 1990

13) MERCK \& CO., INC: The Merck Index. An encyclopedia of chemicals, drugs, and biologicals. Ed., S. Budavari $e t$ al., Merck \& Co., Inc., Rahway, N.J., U.S.A., 1989

14) Siemann, M.; L. I. Andersson \& K. Mosbach: Selective recognition of the herbicide atrazine by noncovalent molecularly imprinted polymers. J. Agric. Food Chem. 44: $141 \sim 145,1996$ 


\section{Cladinose Analogues of Sixteen-membered Macrolide Antibiotics}

\section{Preparation of Pharmacokinetically Improved Analogues via Biotransformation}

\author{
KeIICHI AJito*, Ken-ICHi Kurihara, \\ Seij Shibahara, Osamu Hara, \\ Akira Shimizu, Minako Araake \\ and Sнолг Омото
}

Pharmaceutical Research Center, Meiji Seika Kaisha, Ltd., Morooka-cho, Kohoku-ku, Yokohama 222, Japan

(Received for publication June 20, 1996)

Sixteen-membered macrolide antibiotics ${ }^{1)}$ have been used clinically for many years because of their excellent efficacy and safety. Since, they do not always exhibit satisfactory pharmacokinetics ${ }^{2)}$ compared with fourteenmembered macrolides, the design and synthesis of analogues showing improved pharmacokinetics would be an important achievement from the clinical point of view. As part of our program in this area, we have recently designed, synthesized and studied 4- $O$-alkyl-L-cladinose analogues, compounds $(\mathbf{1} \sim \mathbf{5})$, including 9-dehydro-3"$O$-methyl-4"-O-(3-methylbutyl)-3-O-propionylleucomycin $\mathrm{V}^{3)}$, compound 3 (Scheme 1 ). In this communication, we wish to report the preparation of the potentially more useful C-9 hydroxyl analogues, compounds $\mathbf{6} \sim 10$. The reported compound 8 exhibited dramatically improved pharmacokinetics in mice.

As we reported in our previous paper ${ }^{3)}$, one of the 4- $O$-alkyl-L-cladinose analogues, 3 , showed better pharmacokinetics in mice compared to midecamycin $\mathrm{A}_{3}$ (Fig. 1) having 4-O-acylated sugar moiety. Pharmacokinetic comparison between midecamycin $\mathrm{A}_{1}$ and $\mathrm{A}_{3}$ in mice in vivo ${ }^{4}$ (Fig. 1) led us to reduce a carbonyl group at the C-9 position to generate an $\alpha$-hydroxyl group stereoselectively. As a result of this modification, improvement in both serum concentration and urinary excretion in vivo was achieved. Some methods for reducing the C-9 carbonyl of sixteen-membered macrolides to the alcohol through synthetic approaches ${ }^{5 \sim 7)}$ and biochemical approaches ${ }^{8 \sim 11}$ have been reported. After careful consideration of these methodologies, we chose the biotransformation ${ }^{9,10)}$ of these compounds because of the exclusive stereoselectivity and ease of procedure.

Bioconversion of a C-9 carbonyl to a corresponding $\alpha$-hydroxyl group was successfully completed in spite of the presence of an unnatural sugar (4-O-alkyl-Lcladinose). In general, the medium ${ }^{\dagger}$ was pipetted in $80 \mathrm{ml}$ portions into three $500 \mathrm{ml}$-Erlenmeyer's flasks and sterilized at $120^{\circ} \mathrm{C}$ for 30 minutes. The medium in each flask was inoculated with $1.6 \mathrm{ml}$ of a frozen seed of a non-producing mutant of Streptomyces mycarofaciens SF-837, for example Streptomyces mycarofaciens SF $2772^{4)}$ strain, having a cell density of $10 \sim 15 \%$ which was then incubated at $28^{\circ} \mathrm{C}$ for 24 hours while shaking. Streptomyces mycarofaciens SF 2772 has been deposited with Fermentation Research Institute of agency of Industrial Science and Technology, of 1-3, Higashi 1-chome, Tsukuba-shi, Ibaraki 305, Japan, under the accession number FERM BP-4465. SF2772 strain can be constructed by artificial mutagenesis of the SF- 837 strain by known methods. Streptomyces mycarofaciens SF-837 has been deposited with Fermentation Research Institute of Agency of Industrial Science and Technology under the accession number FERM P-262 and with American Type Culture Collection under the accession number of ATCC21454 and now available to the public.

Scheme 1.

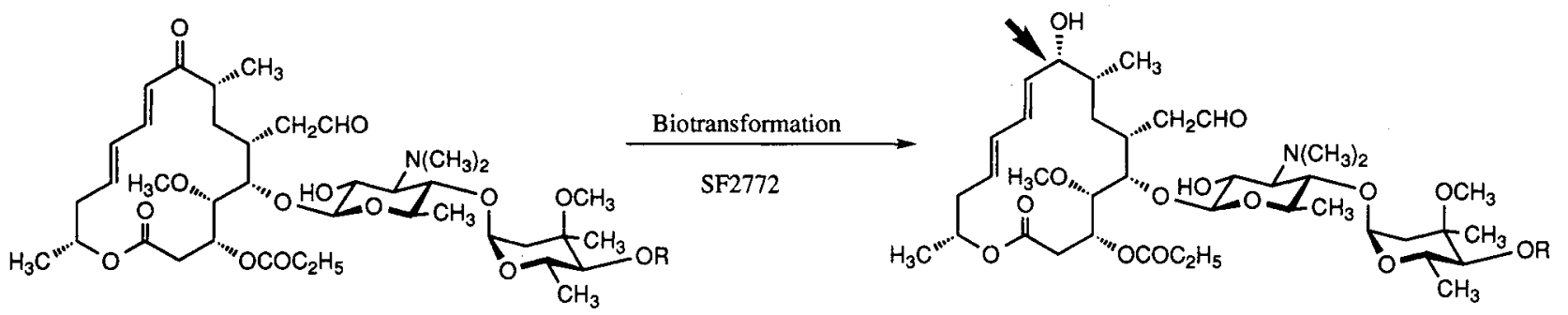

R

$\begin{array}{llr}\mathbf{1} & \mathrm{CH}_{2} \mathrm{CH}=\mathrm{CH}_{2} & 6 \\ 3 & \mathrm{CH}_{2} \mathrm{CH}_{2} \mathrm{CH}_{2} \mathrm{CH}_{3} & 7 \\ 4 & \left.\mathrm{CH}_{2} \mathrm{CH}_{2} \mathrm{CH}_{(} \mathrm{CH}_{3}\right)_{2} & \mathbf{8} \\ 5 & \mathrm{CH}_{2} \mathrm{CH}_{2} \mathrm{CH}_{2} \mathrm{CH}_{2} \mathrm{CH}_{2} \mathrm{CH}_{3} & 9 \\ \mathrm{CH}_{2} \mathrm{C}_{6} \mathrm{H}_{5} & 10\end{array}$

$\uparrow$ A medium comprising $2.0 \%$ of glucose, $1.0 \%$ of polypeptone, $0.05 \%$ of dipotassium hydrogenphosphate, $0.05 \%$ of magnesium sulfate heptahydrate and $0.3 \%$ of sodium chloride was adjusted to $\mathrm{pH} 7.0$ and sterilized prior to the use. 
Fig. 1. Structure of midecamycins.

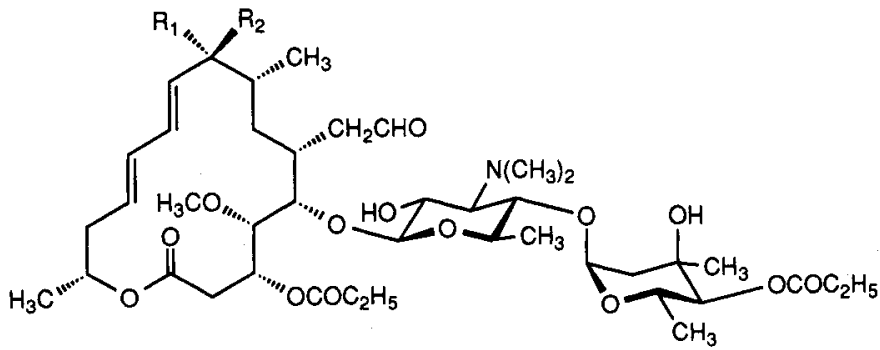

Midecamycin $A_{1} \quad R_{1}=O H, R_{2}=H$

Midecamycin $A_{3} R_{1}, R_{2}=0$

Table 1. Antibacterial activities of $\mathbf{1} \sim \mathbf{1 0}$ and midecamycin $\mathrm{A}_{1}$ (MIC, $\mu \mathrm{g} / \mathrm{ml}$ ).

\begin{tabular}{|c|c|c|c|c|c|c|c|c|c|c|c|}
\hline Test organisms & 1 & 2 & 3 & 4 & 5 & 6 & 7 & 8 & 9 & 10 & $\begin{array}{l}\text { Mideca- } \\
\text { mycin } A_{1}\end{array}$ \\
\hline $\begin{array}{l}\text { Staphylococcus aureus } \\
209 \text { P JC-1 }\end{array}$ & 3.13 & 0.20 & 0.20 & 0.78 & 0.20 & 3.13 & 0.39 & 0.20 & 0.39 & 3.13 & 0.39 \\
\hline S. aureus M133 & 12.5 & 0.78 & 0.78 & 1.56 & 1.56 & 6.25 & 0.78 & 0.78 & 1.56 & 12.5 & 0.78 \\
\hline S. aureus M126 & $>100$ & $>100$ & $>100$ & $>100$ & $>100$ & $>100$ & $>100$ & $>100$ & $>100$ & $>100$ & $>100$ \\
\hline S. aureus MS15026 & $>100$ & $>100$ & $>100$ & $>100$ & $>100$ & $>100$ & $>100$ & $>100$ & $>100$ & $>100$ & $>100$ \\
\hline S. aureus MS15027 & 12.5 & 0.78 & 0.78 & 1.56 & 1.56 & 6.25 & 0.78 & 0.78 & 1.56 & 6.25 & 0.78 \\
\hline S. epidermidis ATCC 14990 & 25 & 1.56 & 0.78 & 1.56 & 3.13 & 12.5 & 1.56 & 1.56 & 1.56 & 25 & 1.56 \\
\hline $\begin{array}{l}\text { Micrococcus luteus } \\
\text { ATCC } 9341\end{array}$ & 0.39 & 0.05 & 0.05 & 0.10 & 0.10 & 0.39 & 0.05 & 0.05 & 0.10 & 0.78 & 0.05 \\
\hline Enterococcus faecalis W-73 & 6.25 & 3.13 & 1.56 & 6.25 & 1.56 & 6.25 & 3.13 & 3.13 & 3.13 & 6.25 & 3.13 \\
\hline $\begin{array}{l}\text { Streptococcus pneumoniae } \\
\text { IP692 }\end{array}$ & 1.56 & 0.10 & 0.10 & 0.20 & 0.20 & 0.78 & 0.05 & 0.10 & 0.20 & 0.78 & 0.39 \\
\hline S. pneumoniae Type $\mathbf{I}$ & 1.56 & 0.20 & 0.20 & 0.20 & 0.20 & 0.78 & 0.20 & 0.20 & 0.20 & 1.56 & 0.39 \\
\hline S. pyogenes Cook & 1.56 & 0.10 & 0.05 & 0.20 & 0.20 & 0.78 & 0.20 & 0.10 & 0.20 & 0.78 & 0.20 \\
\hline Escherichia coli NIHJ JC-2 & $>100$ & $>100$ & $>100$ & $>100$ & $>100$ & $>100$ & $>100$ & $>100$ & $>100$ & $>100$ & $>100$ \\
\hline $\begin{array}{l}\text { Klebsiella pneumoniae } \\
\text { PCI } 602\end{array}$ & $>100$ & $>100$ & $>100$ & $>100$ & $>100$ & $>100$ & $>100$ & $>100$ & $>100$ & $>100$ & $>100$ \\
\hline $\begin{array}{l}\text { Branhamella catarrhalis } \\
\text { W-0500 }\end{array}$ & 12.5 & 0.78 & 0.78 & 1.56 & 1.56 & 6.25 & 1.56 & 0.78 & 1.56 & 6.25 & 3.13 \\
\hline B. catarrhalis W-0506 & 6.25 & 0.78 & 0.78 & 1.56 & 1.56 & 6.25 & 1.56 & 1.56 & 1.56 & 6.25 & 1.56 \\
\hline Haemophilus influenzae 9334 & NT & NT & NT & NT & NT & 50 & 6.25 & 6.25 & 6.25 & 50 & 3.13 \\
\hline Mycoplasma pneumoniae $\mathrm{FH}$ & NT & NT & NT & NT & NT & NT & 1.56 & 1.56 & 1.56 & NT & 1.56 \\
\hline M. pneumoniae $\mathrm{Mac}$ & NT & NT & NT & NT & NT & $\mathrm{NT}$ & 0.10 & 0.05 & 0.20 & NT & 0.10 \\
\hline M. pneumoniae FH-P24 & NT & NT & NT & NT & NT & NT & 0.10 & 0.025 & 0.20 & N'T & 0.10 \\
\hline M. pneumoniae Numata & NT & NT & NT & NT & NT & NT & 0.05 & 0.025 & 0.05 & NT & 0.05 \\
\hline
\end{tabular}

NT; Not tested.

Next, $1.2 \mathrm{ml}$ of a methanol solution containing $20 \mathrm{mg}$ of the carbonyl compound was added to each flask in $0.4 \mathrm{ml}$ portions and the incubation was continued at $28^{\circ} \mathrm{C}$ for 17 hours while shaking. After the completion of the incubation, the culture was centrifuged at $3000 \mathrm{rpm}$ for 10 minutes. Thus $180 \mathrm{ml}$ of a transparent culture supernatant was obtained while the solid matters including the cells were removed. To the solid matters was added $120 \mathrm{ml}$ of water and the mixture was stirred followed by centrifugation. The washing liquor thus obtained was combined with the above-mentioned transparent culture supernatant. After adjusting the mixture to $\mathrm{pH} 9$ with a $1 \mathrm{~N}$ aqueous solution of sodium hydroxide, the conversion product was extracted with $300 \mathrm{ml}$ portions of ethyl acetate twice. The ethyl acetate layer was dried over anhydrous sodium sulfate and then filtered. The filtrate was concentrated under reduced pressure and the residue thus obtained was purified by preparative TLC [developing system : chloroform/methanol $(10: 1)]$. Thus, $9 \sim 12 \mathrm{mg}(45 \sim 60 \%)$ of the desired $\alpha$-hydroxyl compounds were obtained with trace of a starting material. For example, evidence of the desired $\alpha$-hydroxyl stereochemistry for compound $\mathbf{8}$ was demonstrated by ${ }^{1} \mathrm{H}$ NMR experiments $(\delta 4.07(1 \mathrm{H}$, dd, $\left.\left.J_{8,9}=4.0 \mathrm{~Hz}, J_{9,10}=10.0 \mathrm{~Hz}, 9-\mathrm{H}\right)\right)^{6}$.

The antibacterial activities in vitro of novel 4-O-alkylL-cladinosyl derivatives $(\mathbf{6} \sim 10)$, compared with corresponding midecamycin $A_{1}$, are shown in Table $1^{\dagger \dagger}$. Although compounds 7 and $\mathbf{8}$ displayed similar in vitro activities to midecamycin $\mathrm{A}_{1}$, their activities against Streptococcus pneumoniae were clearly enhanced compared with midecamycin $A_{1}$. The activity of $\mathbf{8}$ against Mycoplasma pneumoniae was also quite high. As expected, stereoselective reduction at the C-9 position of 9-keto derivatives did not reduce their original antibacterial activities.

${ }_{\dagger}$ All biological studies were done as described in our previous paper. See ref. 3. 
Table 2. Physico-chemical properties of $\mathbf{6} \sim \mathbf{1 0}$.

Compound (6): EI-MS $m / z 811(\mathrm{M})^{+} ;[\alpha]_{\mathrm{D}}-55^{\circ}\left(\mathrm{c} 0.6, \mathrm{CH}_{3} \mathrm{OH}\right) ; \mathrm{MP} 101 \sim 106^{\circ} \mathrm{C} ;{ }^{1} \mathrm{H} \mathrm{NMR}\left(400 \mathrm{MHz}, \mathrm{CDCl}_{3}\right) \delta 0.99(3 \mathrm{H}$, d, 19-H), $1.16\left(3 \mathrm{H}, \mathrm{d}, 6^{\prime}-\mathrm{H}\right), 1.22\left(3 \mathrm{H}, \mathrm{t}, 3-\mathrm{OCOCH}_{2} \mathrm{CH}_{3}\right), 1.23\left(3 \mathrm{H}, \mathrm{d}, 6^{\prime \prime}-\mathrm{H}\right), 1.24\left(3 \mathrm{H}, \mathrm{s}, 3^{\prime \prime}-\mathrm{CH}_{3}\right), 1.26(3 \mathrm{H}, \mathrm{d}, 16-\mathrm{H}), 1.57$ $\left(1 \mathrm{H}, \mathrm{dd}, 2^{\prime \prime}-\mathrm{Hax}\right), 1.89(1 \mathrm{H}, \mathrm{m}, 8-\mathrm{H}), 2.24(1 \mathrm{H}, \mathrm{brd}, 2-\mathrm{H}), 2.24\left(1 \mathrm{H}, \mathrm{d}, 2^{\prime \prime}-\mathrm{Heq}\right), 2.51$ and 2.64 (each $\left.2 \mathrm{H}, \mathrm{dq}, 3-\mathrm{OCOCH}_{2} \mathrm{CH}_{3}\right)$, $2.65\left(6 \mathrm{H}, \mathrm{s}, 3^{\prime}-\mathrm{N}\left(\mathrm{CH}_{3}\right)_{2}\right), 2.76(1 \mathrm{H}, \mathrm{dd}, 2-\mathrm{H}), 2.84(1 \mathrm{H}, \mathrm{brdd}, 17-\mathrm{H}), 2.87\left(1 \mathrm{H}, \mathrm{d}, 4^{\prime \prime}-\mathrm{H}\right), 3.25\left(3 \mathrm{H}, \mathrm{s}, 3^{\prime \prime}-\mathrm{OCH}_{3}\right), 3.26(1 \mathrm{H}, \mathrm{brd}$, 4-H), $3.28\left(1 \mathrm{H}, \mathrm{dq}, 5^{\prime}-\mathrm{H}\right), 3.50\left(1 \mathrm{H}, \mathrm{t}, 4^{\prime}-\mathrm{H}\right), 3.57\left(3 \mathrm{H}, \mathrm{s}, 4-\mathrm{OCH}_{3}\right), 3.87(1 \mathrm{H}, \mathrm{br} \mathrm{d}, 5-\mathrm{H}), 4.07(1 \mathrm{H}, \mathrm{dd}, 9-\mathrm{H}), 4.11$ and 4.19 (each $2 \mathrm{H}$, brdd, 4"-OCH $\left.\mathrm{H}_{2} \mathrm{CH}=\mathrm{CH}_{2}\right), 4.40\left(1 \mathrm{H}, \mathrm{dq}, 5^{\prime \prime}-\mathrm{H}\right), 4.54\left(1 \mathrm{H}, \mathrm{d}, \mathrm{l}^{\prime}-\mathrm{H}\right), 4.91\left(1 \mathrm{H}, \mathrm{d}, 1^{\prime \prime}-\mathrm{H}\right), 5.03(1 \mathrm{H}, \mathrm{ddq}, 15-\mathrm{H}), 5.13(1 \mathrm{H}, \mathrm{brd}$, $3-\mathrm{H}), 5.17$ and $5.23\left(\right.$ each $2 \mathrm{H}$, brd, $\left.4^{\prime \prime}-\mathrm{OCH}_{2} \mathrm{CH}=\mathrm{CH}_{2}\right), 5.62(1 \mathrm{H}, \mathrm{dd}, 10-\mathrm{H}), 5.79(1 \mathrm{H}, \mathrm{ddd}, 13-\mathrm{H}), 5.95(1 \mathrm{H}$, ddt, 4"- $\left.\mathrm{OCH}_{2} \mathrm{CH}=\mathrm{CH}_{2}\right), 6.08(1 \mathrm{H}$, br dd, $12-\mathrm{H}), 6.68(1 \mathrm{H}, \mathrm{dd}, 11-\mathrm{H}), 9.63(1 \mathrm{H}, \mathrm{s}, 18-\mathrm{H})$.

Compound (7): EI-MS $m / z 827(\mathrm{M})^{+} ;[\alpha]_{\mathrm{D}}-50^{\circ}\left(c 0.9, \mathrm{CH}_{3} \mathrm{OH}\right) ; \mathrm{MP} 99 \sim 101^{\circ} \mathrm{C} ;{ }^{1} \mathrm{H} \mathrm{NMR}\left(400 \mathrm{MHz}, \mathrm{CDCl}_{3}\right) \delta 0.91(3 \mathrm{H}, \mathrm{t}$, $\left.4^{\prime \prime}-\mathrm{OCH}_{2} \mathrm{CH}_{2} \mathrm{CH}_{2} \mathrm{CH}_{3}\right), 0.98(3 \mathrm{H}, \mathrm{d}, 19-\mathrm{H}), 1.15\left(3 \mathrm{H}, \mathrm{d}, 6^{\prime}-\mathrm{H}\right), 1.21\left(3 \mathrm{H}, \mathrm{t}, 3-\mathrm{OCOCH}_{2} \mathrm{CH}_{3}\right), 1.22\left(3 \mathrm{H}, \mathrm{d}, 6^{\prime \prime}-\mathrm{H}\right), 1.24(3 \mathrm{H}, \mathrm{s}$, $\left.3^{\prime \prime}-\mathrm{CH}_{3}\right), 1.26(3 \mathrm{H}, \mathrm{d}, 16-\mathrm{H}), 1.37\left(2 \mathrm{H}, \mathrm{m}, 4^{\prime \prime}-\mathrm{OCH}_{2} \mathrm{CH}_{2} \mathrm{CH}_{2} \mathrm{CH}_{3}\right), 1.57\left(1 \mathrm{H}, \mathrm{dd}, 2^{\prime \prime}-\mathrm{Hax}\right), 1.60\left(2 \mathrm{H}, \mathrm{m}, 4^{\prime \prime}-\mathrm{OCH}_{2} \mathrm{CH}_{2} \mathrm{CH}_{2} \mathrm{CH}_{3}\right)$, $1.89(1 \mathrm{H}, \mathrm{m}, 8-\mathrm{H}), 2.23\left(1 \mathrm{H}, \mathrm{d}, 2^{\prime \prime}-\mathrm{Heq}\right), 2.24(1 \mathrm{H}, \mathrm{brd}, 2-\mathrm{H}), 2.51$ and 2.64 (each $\left.2 \mathrm{H}, \mathrm{dq}, 3-\mathrm{OCOCH}_{2} \mathrm{CH}_{3}\right), 2.62(6 \mathrm{H}, \mathrm{s}$, $\left.3^{\prime}-\mathrm{N}\left(\mathrm{CH}_{3}\right)_{2}\right), 2.76(1 \mathrm{H}, \mathrm{dd}, 2-\mathrm{H}), 2.78\left(1 \mathrm{H}, \mathrm{d}, 4^{\prime \prime}-\mathrm{H}\right), 2.85\left(1 \mathrm{H}\right.$, br dd, 17-H), $3.22\left(1 \mathrm{H}\right.$, br dd, $\left.2^{\prime}-\mathrm{H}\right), 3.25(1 \mathrm{H}$, br d, $4-\mathrm{H})$, $3.25\left(3 \mathrm{H}, \mathrm{s}, 3^{\prime \prime}-\mathrm{OCH}_{3}\right), 3.28\left(1 \mathrm{H}, \mathrm{dq}, 5^{\prime}-\mathrm{H}\right), 3.48\left(1 \mathrm{H}, \mathrm{t}, 4^{\prime}-\mathrm{H}\right), 3.57\left(3 \mathrm{H}, \mathrm{s}, 4-\mathrm{OCH}_{3}\right), 3.57$ and, 3.62 (each $2 \mathrm{H}, \mathrm{dt}$, 4"-OCH $\left.\mathrm{CH}_{2} \mathrm{CH}_{2} \mathrm{CH}_{3}\right), 3.87\left(1 \mathrm{H}\right.$, br d, 5-H), $4.07(1 \mathrm{H}, \mathrm{dd}, 9-\mathrm{H}), 4.39\left(1 \mathrm{H}, \mathrm{dq}, 5^{\prime \prime}-\mathrm{H}\right), 4.53\left(1 \mathrm{H}, \mathrm{d}, \mathrm{l}^{\prime}-\mathrm{H}\right), 4.89\left(1 \mathrm{H}, \mathrm{d}, \mathrm{l}^{\prime \prime}-\mathrm{H}\right)$, $5.03(1 \mathrm{H}, \mathrm{ddq}, 15-\mathrm{H}), 5.13(1 \mathrm{H}, \mathrm{brd}, 3-\mathrm{H}), 5.62(1 \mathrm{H}, \mathrm{dd}, 10-\mathrm{H}), 5.79(1 \mathrm{H}, \mathrm{ddd}, 13-\mathrm{H}), 6.08(1 \mathrm{H}, \mathrm{brdd}, 12-\mathrm{H}), 6.67(1 \mathrm{H}, \mathrm{dd}$, $11-\mathrm{H}), 9.63(1 \mathrm{H}, \mathrm{s}, 18-\mathrm{H})$

Compound (8): EI-MS $m / z 841(\mathrm{M})^{+} ;[\alpha]_{\mathrm{D}}-49^{\circ}\left(c 0.7, \mathrm{CH}_{3} \mathrm{OH}\right) ; \mathrm{MP} 98 \sim 100^{\circ} \mathrm{C} ;{ }^{1} \mathrm{H}$ NMR $\left(400 \mathrm{MHz}, \mathrm{CDCl}_{3}\right) \delta 0.89(6 \mathrm{H}$, $\left.\mathrm{d}, 4^{\prime \prime}-\mathrm{OCH}_{2} \mathrm{CH}_{2} \mathrm{CH}\left(\mathrm{CH}_{3}\right)_{2}\right), 0.99(3 \mathrm{H}, \mathrm{d}, 19-\mathrm{H}), 1.15\left(3 \mathrm{H}, \mathrm{d}, 6^{\prime}-\mathrm{H}\right), 1.22\left(3 \mathrm{H}, \mathrm{t}, 3-\mathrm{OCOCH}_{2} \mathrm{CH}_{3}\right), 1.23\left(3 \mathrm{H}, \mathrm{d}, 6^{\prime \prime}-\mathrm{H}\right), 1.24(3 \mathrm{H}$, s, 3"- $\left.\mathrm{CH}_{3}\right), 1.26(3 \mathrm{H}, \mathrm{d}, 16-\mathrm{H}), 1.57\left(1 \mathrm{H}, \mathrm{dd}, 2^{\prime \prime}-\mathrm{Hax}\right), 1.69\left(1 \mathrm{H}, \mathrm{m}, 4^{\prime \prime}-\mathrm{OCH}_{2} \mathrm{CH}_{2} \mathrm{CH}\left(\mathrm{CH}_{3}\right)_{2}\right), 1.89(1 \mathrm{H}, \mathrm{m}, 8-\mathrm{H}), 2.22(1 \mathrm{H}, \mathrm{d}$, $\left.2^{\prime \prime}-\mathrm{Heq}\right), 2.24(1 \mathrm{H}, \mathrm{brd}, 2-\mathrm{H}), 2.51$ and $2.64\left(\right.$ each $\left.2 \mathrm{H}, \mathrm{dq}, 3-\mathrm{OCOCH}_{2} \mathrm{CH}_{3}\right), 2.62\left(6 \mathrm{H}, \mathrm{s}, 3^{\prime}-\mathrm{N}\left(\mathrm{CH}_{3}\right)_{2}\right), 2.76(1 \mathrm{H}, \mathrm{dd}, 2-\mathrm{H}), 2.78$ $\left(1 \mathrm{H}, \mathrm{d}, 4^{\prime \prime}-\mathrm{H}\right), 2.85\left(1 \mathrm{H}\right.$, br dd, 17-H), $3.22\left(1 \mathrm{H}\right.$, br dd, $\left.2^{\prime}-\mathrm{H}\right), 3.25\left(3 \mathrm{H}, \mathrm{s}, 3^{\prime \prime}-\mathrm{OCH}_{3}\right), 3.26(1 \mathrm{H}$, br d, $4-\mathrm{H}), 3.28\left(1 \mathrm{H}, \mathrm{dq}, 5^{\prime}-\mathrm{H}\right)$, $3.48\left(1 \mathrm{H}, \mathrm{t}, 4^{\prime}-\mathrm{H}\right), 3.57\left(3 \mathrm{H}, \mathrm{s}, 4-\mathrm{OCH}_{3}\right), 3.60$ and 3.64 (each $\left.2 \mathrm{H}, \mathrm{dt}, 4^{\prime \prime}-\mathrm{OCH} \mathrm{CH}_{2} \mathrm{CH}\left(\mathrm{CH}_{3}\right)_{2}\right), 3.87(1 \mathrm{H}$, br d, $5-\mathrm{H}), 4.07(1 \mathrm{H}$, dd, 9-H), $4.39\left(1 \mathrm{H}, \mathrm{dq}, 5^{\prime \prime}-\mathrm{H}\right), 4.53\left(1 \mathrm{H}, \mathrm{d}, \mathrm{l}^{\prime}-\mathrm{H}\right), 4.89\left(1 \mathrm{H}, \mathrm{d}, \mathrm{l}^{\prime \prime}-\mathrm{H}\right), 5.03(1 \mathrm{H}, \mathrm{ddq}, 15-\mathrm{H}), 5.13(1 \mathrm{H}, \mathrm{brd}, 3-\mathrm{H}), 5.62(1 \mathrm{H}, \mathrm{dd}$, $10-\mathrm{H}), 5.79(1 \mathrm{H}, \mathrm{ddd}, 13-\mathrm{H}), 6.08(1 \mathrm{H}, \mathrm{brdd}, 12-\mathrm{H}), 6.68(1 \mathrm{H}, \mathrm{dd}, 11-\mathrm{H}), 9.63(1 \mathrm{H}, \mathrm{s}, 18-\mathrm{H})$.

Compound (9): EI-MS $m / z 855(\mathrm{M})^{+} ;[\alpha]_{\mathrm{D}}-50^{\circ}\left(c 0.8, \mathrm{CH}_{3} \mathrm{OH}\right) ; \mathrm{MP} 96 \sim 102^{\circ} \mathrm{C} ;{ }^{1} \mathrm{H} \mathrm{NMR}\left(400 \mathrm{MHz}, \mathrm{CDCl}_{3}\right) \delta 0.88(3 \mathrm{H}, \mathrm{t}$, 4"- $\left.\mathrm{OCH}_{2} \mathrm{CH}_{2}\left(\mathrm{CH}_{2}\right)_{3} \mathrm{CH}_{3}\right), 0.99(3 \mathrm{H}, \mathrm{d}, 19-\mathrm{H}), 1.15\left(3 \mathrm{H}, \mathrm{d}, 6^{\prime}-\mathrm{H}\right), 1.22\left(3 \mathrm{H}, \mathrm{t}, 3-\mathrm{OCOCH}_{2} \mathrm{CH}_{3}\right), 1.23\left(3 \mathrm{H}, \mathrm{d}, 6^{\prime \prime}-\mathrm{H}\right), 1.24(3 \mathrm{H}, \mathrm{s}$, $\left.3^{\prime \prime}-\mathrm{CH}_{3}\right), 1.26(3 \mathrm{H}, \mathrm{d}, 16-\mathrm{H}), 1.57\left(1 \mathrm{H}, \mathrm{dd}, 2^{\prime \prime}-\mathrm{Hax}\right), 1.61\left(2 \mathrm{H}, \mathrm{m}, 4^{\prime \prime}-\mathrm{OCH}_{2} \mathrm{CH}_{2}\left(\mathrm{CH}_{2}\right)_{3} \mathrm{CH}_{3}\right), 1.89(1 \mathrm{H}, \mathrm{m}, 8-\mathrm{H}), 2.23(1 \mathrm{H}, \mathrm{d}$, $\left.2^{\prime \prime}-\mathrm{Heq}\right), 2.24(1 \mathrm{H}, \mathrm{brd}, 2-\mathrm{H}), 2.5 \mathrm{l}$ and 2.64 (each $\left.2 \mathrm{H}, \mathrm{dq}, 3-\mathrm{OCOCH}_{2} \mathrm{CH}_{3}\right), 2.63\left(6 \mathrm{H}, \mathrm{s}, 3^{\prime}-\mathrm{N}\left(\mathrm{CH}_{3}\right)_{2}\right), 2.76(1 \mathrm{H}, \mathrm{dd}, 2-\mathrm{H}), 2.78$ $\left(1 \mathrm{H}, \mathrm{d}, 4^{\prime \prime}-\mathrm{H}\right), 2.85(1 \mathrm{H}$, br dd, $17-\mathrm{H}), 3.22\left(1 \mathrm{H}\right.$, br dd, $\left.2^{\prime}-\mathrm{H}\right), 3.25(1 \mathrm{H}, \mathrm{brd}, 4-\mathrm{H}), 3.25\left(3 \mathrm{H}, \mathrm{s}, 3^{\prime \prime}-\mathrm{OCH}_{3}\right), 3.28\left(1 \mathrm{H}, \mathrm{dq}, 5^{\prime}-\mathrm{H}\right)$, $3.48\left(1 \mathrm{H}, \mathrm{t}, 4^{\prime}-\mathrm{H}\right), 3.55$ and 3.61 (each $\left.2 \mathrm{H}, \mathrm{dt}, 4^{\prime \prime}-\mathrm{OCH}_{2} \mathrm{CH}_{2}\left(\mathrm{CH}_{2}\right)_{3} \mathrm{CH}_{3}\right), 3.57\left(3 \mathrm{H}, \mathrm{s}, 4-\mathrm{OCH}_{3}\right), 3.87(1 \mathrm{H}$, br d, $5-\mathrm{H}), 4.07(1 \mathrm{H}$, dd, 9-H), $4.39\left(1 \mathrm{H}, \mathrm{dq}, 5^{\prime \prime}-\mathrm{H}\right), 4.53\left(1 \mathrm{H}, \mathrm{d}, \mathrm{l}^{\prime}-\mathrm{H}\right), 4.89\left(1 \mathrm{H}, \mathrm{d}, \mathrm{l}^{\prime \prime}-\mathrm{H}\right), 5.03(1 \mathrm{H}, \mathrm{ddq}, 15-\mathrm{H}), 5.13(1 \mathrm{H}, \mathrm{br}, 3-\mathrm{H}), 5.62(1 \mathrm{H}, \mathrm{dd}$, $10-\mathrm{H}), 5.79(1 \mathrm{H}, \mathrm{ddd}, 13-\mathrm{H}), 6.08(1 \mathrm{H}$, br dd, 12-H), $6.68(1 \mathrm{H}, \mathrm{dd}, 11-\mathrm{H}), 9.63(1 \mathrm{H}, \mathrm{s}, 18-\mathrm{H})$.

Compound (10): SI-MS $m / z 862(\mathrm{M}+\mathrm{H})^{+} ;[\alpha]_{\mathrm{D}}-52^{\circ}\left(c 0.8, \mathrm{CH}_{3} \mathrm{OH}\right) ; \mathrm{MP} 112 \sim 116^{\circ} \mathrm{C} ;{ }^{1} \mathrm{H}$ NMR $\left(400 \mathrm{MHz}, \mathrm{CDCl}_{3}\right) \delta$ $0.98(3 \mathrm{H}, \mathrm{d}, 19-\mathrm{H}), 1.15\left(3 \mathrm{H}, \mathrm{d}, 6^{\prime}-\mathrm{H}\right), 1.15\left(3 \mathrm{H}, \mathrm{s}, 3^{\prime \prime}-\mathrm{CH}_{3}\right), 1.21\left(3 \mathrm{H}, \mathrm{t}, 3-\mathrm{OCOCH}_{2} \mathrm{CH}_{3}\right), 1.23\left(3 \mathrm{H}, \mathrm{d}, 6^{\prime \prime}-\mathrm{H}\right), 1.26(3 \mathrm{H}, \mathrm{d}$, 16-H), $1.57\left(1 \mathrm{H}, \mathrm{dd}, 2^{\prime \prime}-\mathrm{Hax}\right), \mathrm{I} .89(\mathrm{HH}, \mathrm{m}, 8-\mathrm{H}), 2.22\left(1 \mathrm{H}, \mathrm{d}, 2^{\prime \prime}-\mathrm{Heq}\right), 2.24(1 \mathrm{H}, \mathrm{brd}, 2-\mathrm{H}) ; 2.31(1 \mathrm{H}, \mathrm{brdd}, 17-\mathrm{H}), 2.51$ and 2.64 (each $\left.2 \mathrm{H}, \mathrm{dq}, 3-\mathrm{OCOCH}_{2} \mathrm{CH}_{3}\right), 2.62\left(6 \mathrm{H}, \mathrm{s}, 3^{\prime}-\mathrm{N}\left(\mathrm{CH}_{3}\right)_{2}\right), 2.76(1 \mathrm{H}, \mathrm{dd}, 2-\mathrm{H}), 2.84(1 \mathrm{H}$, br dd, $17-\mathrm{H}), 3.00\left(1 \mathrm{H}, \mathrm{d}, 4^{\prime \prime}-\mathrm{H}\right)$, $3.25(1 \mathrm{H}$, brd, $4-\mathrm{H}), 3.25\left(3 \mathrm{H}, \mathrm{s}, 3^{\prime \prime}-\mathrm{OCH}_{3}\right), 3.28\left(1 \mathrm{H}, \mathrm{dq}, 5^{\prime}-\mathrm{H}\right), 3.49\left(1 \mathrm{H}, \mathrm{t}, 4^{\prime}-\mathrm{H}\right), 3.57\left(3 \mathrm{H}, \mathrm{s}, 4-\mathrm{OCH}_{3}\right), 3.87(1 \mathrm{H}, \mathrm{brd}, 5-\mathrm{H})$, $4.07(1 \mathrm{H}, \mathrm{dd}, 9-\mathrm{H}), 4.45\left(1 \mathrm{H}, \mathrm{brdq}, 5^{\prime \prime}-\mathrm{H}\right), 4.54\left(1 \mathrm{H}, \mathrm{d}, \mathrm{1}^{\prime}-\mathrm{H}\right), 4.62$ and $4.70\left(\mathrm{each} 2 \mathrm{H}, \mathrm{d}, 4^{\prime \prime}-\mathrm{OCH}_{2} \mathrm{C}_{6} \mathrm{H}_{5}\right), 4.90\left(1 \mathrm{H}, \mathrm{d}, 1^{\prime \prime}-\mathrm{H}\right)$, $5.03(1 \mathrm{H}, \mathrm{ddq}, 15-\mathrm{H}), 5.13(1 \mathrm{H}$, br d, 3-H), $5.62(1 \mathrm{H}, \mathrm{dd}, 10-\mathrm{H}), 5.79(1 \mathrm{H}, \mathrm{ddd}, 13-\mathrm{H}), 6.08(1 \mathrm{H}, \mathrm{br} d \mathrm{~d}, 12-\mathrm{H}), 6.68(1 \mathrm{H}, \mathrm{dd}$, $11-\mathrm{H}), 7.3 \sim 7.4\left(5 \mathrm{H}, \mathrm{m}, 4^{\prime \prime}-\mathrm{OCH}_{2} \mathrm{C}_{6} H_{5}\right), 9.63(1 \mathrm{H}, \mathrm{s}, 18-\mathrm{H})$.

These compounds $(7 \sim 9)$ also exhibited good metabolic stability in rat plasma in vitro. After 24 hours of incubation $^{+\dagger}, 71,85$, and $79 \%$ of initial activity $(\mathrm{t}=0)$ was observed against Micrococcus luteus for compound $\mathbf{7}, \mathbf{8}$, and $\mathbf{9}$, respectively, while only $2 \%$ activity remained for midecamycin $\mathrm{A}_{1}$. Thus, these 4- $O$-alkyl-L-cladinosyl analogues proved to be more stable than midecamycin $\mathrm{A}_{1}$ in vitro. Improved stability is best explained by inability of neutral sugars to be metabolized by esterase $^{12)}$.

Compound (8), which was the best compound in terms of potency and stability, was finally examined in vivo. Serum concentrations of antibiotics in mice after 200 $\mathrm{mg} / \mathrm{kg}$ oral administration ${ }^{\dagger \dagger \dagger}$ are shown in Fig. 2. Serum concentration of $\mathbf{8}$ was dramatically higher and longer lasting than midecamycin $\mathrm{A}_{1}$ and its $\mathrm{AUC}$ was also greater than that of midecamycin $A_{1}$. The maximum concentration of $\mathbf{8}$ in serum was comparable to that of

i+ A test compound was mixed with a $0.2 \%$ aqueous solution of CMC to give a concentration of $4.0 \mathrm{mg} / \mathrm{ml}$ and a $1.0 \mathrm{ml}$ portion of the resulting emulsion was orally administered to 4 weeks old male Jcl: ICR mice. Blood was collected from the armpits of the mice $0.5,1,2,4$ and 6 hours after the administration of the test compound $(n=2)$. The collected blood was allowed to stand at $0^{\circ} \mathrm{C}$ for 2 hours and centrifuged at $3000 \mathrm{rpm}$ for 20 minutes to obtain serum. To the serum was added an equivalent volume of $50 \% \mathrm{CH}_{3} \mathrm{CN}-0.05 \mathrm{M}$ phosphate buffer ( $\mathrm{pH} \mathrm{7.0)}$. The resulting mixture served as a serum sample. The concentration of the test compound in the serum sample was measured by a bioassay method using $M$. luteus ATCC 9341. 
Fig. 2. Concentration in serum.

$200 \mathrm{mg} / \mathrm{kg}$, mouse, $\mathrm{n}=2$, p.o., $\bigcirc \mathbf{8}$, $\bullet$ midecamycin $\mathrm{A}_{1}$, clarithromycin.

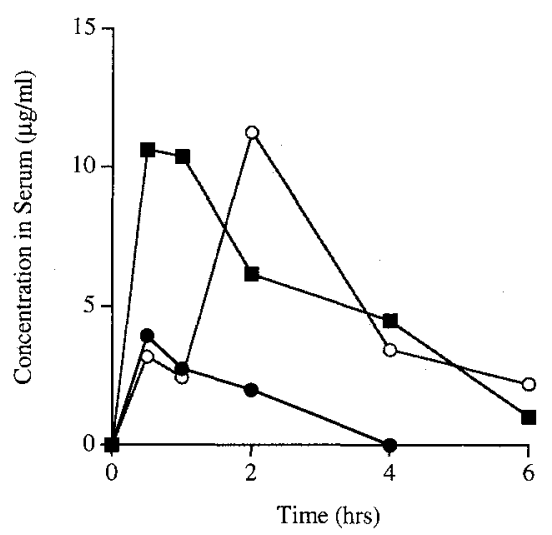

clarithromycin. Moreover urinary recovery of $\mathbf{8}$ within 24 hours in mice $(200 \mathrm{mg} / \mathrm{kg} \text {, p.o. })^{\dagger \dagger \dagger}$ was $20 \%$, while less than $1 \%$ for midecamycin $\mathrm{A}_{1}$, but $24 \%$ for clarithromycin. These excellent pharmacokinetics of $\mathbf{8}$ could be mainly explained by metabolic stability of the dialkyl neutral sugar.

These results demonstrate the possibility to design and synthesize sixteen-membered macrolide derivatives exhibiting similar efficacy to the second generation fourteen-membered macrolides, so-called new macrolides such as clarithromycin. They also point the way for discovery of highly potent and pharmacokinetically excellent derivatives in the sixteen-membered macrolide fields.

\section{Acknowledgments}

We wish to thank Drs. S. INOUYE and S. Gomi for valuable discussions and encouragement during this study. We are also grateful to Dr. T. IshIzUKA for metabolic stability tests in rat plasma and Miss. N. KIKUCHI for her technical assistance.

\section{References}

1) Ōmura, S. (Ed.): Macrolide Antibiotics. Chemistry, Biology, and Practice. Academic Press Inc., 1984

2) Окамото, R.; T. Fukumoto, K. Imafuku, T. Okubo, K. Kiyoshima, A. Takamatsu \& T. TakeUChI: Screening for 16-membered macrolide-transforming microorganisms. J. Ferment. Technol. 57: 519 528, 1979

3) Kurihara, K.; K. Ajito, S. Shibahara, T. Ishizuka, O. Hara, M. Araake \& S. Omoto: Cladinose analogues of sixteen-membered macrolide antibiotics. I. Synthesis of 4- $O$-alkyl-L-cladinose analogues via glycosylation. J. Antibiotics 49: 582 592, 1996

4) Ajito, K.; K. Kurihara, A. Shimizu, S. Gomi, N. Kikuchi, M. AraAKe, T. Ishizuka, A. Miyata, O. Hara \& S. Shibahara (Meiji Seika Kaisha, LTD.): 16-Membered macrolide derivatives and process for producing the same. United States Patent 5,407,918, Apr. 18, 1995

5) FreiberG, L. A.; R. S. Egan \& W. H. Washburn: The synthesis of 9-epi-leucomycin $\mathrm{A}_{3}$. The revised configurational assignment of $\mathbf{C}-9$ in natural leucomycin $\mathbf{A}_{3}$. $\mathbf{J}$. Org. Chem. 39: 2474 2475, 1974

6) Ōmura, S.; H. Sakakibara, T. Fujiwara \& M. Aizawa: 9-epi-Leucomycin $\mathrm{A}_{5}$ synthesis and antimicrobial activity. J. Antibiotics 34: $1577 \sim 1580,1981$

7) Tsuzuki, K.; H. Matsubara, A. Nakagawa \& S. Ōmura: Synthesis and antimicrobial activities of 9-O-acyl derivatives of tylosin and demycarosyltylosin. J. Antibiotics 39: $1784 \sim 1787,1986$

8) Suzuki, M.; N. Nagahama, Y. Seki \& T. Yamaguchi (Tanabe Seiyaku Co., Ltd.), Jpn. Kokai 126880 ('75), Oct. 6, 1975

9) Nagaoka, K.; H. Ogawa, Y. Matsuhashi, K. Watanabe, T. Matsunobu, T. Shomura \& H. GoI (Meiji Seika Kaisha., Ltd.), Jpn. Kokai 8793 ('79), Jan. 23, 1979

10) Matsuhashi, Y.; H. Ogawa \& K. Nagaoka: The enzymatic interconversion between midecamycin $\mathrm{A}_{1}$ and $\mathrm{A}_{3}$. J. Antibiotics 37: $777 \sim 779,1979$

11) Ōmura, S.; C. Kitao \& N. Sadakane: The microbial transformation of tylosin by the spiramycun-producing strain, Streptomyces ambofaciens KA-1028. J. Antibiotics 33: $911 \sim 912,1980$

12) Shomura, T.; S. Someya, K. Umemura, M. Nishio \& S. Murata: Metabolism of 9,3"-diacetylmidecamycin. I. The metabolic fate of 9,3"-diacetylmidecamycin. Yakugaku Zasshi (Japanese) 102: 781 795, 1982

${ }^{+\dagger \dagger}$ Subsequently, $200 \mathrm{mg} / \mathrm{kg}$ of a test compound was orally administered to three mice in the same manner as described above. The mice were put in a metabolic cage MM type (Sugiyamagen Co., Tokyo, Japan) and urine was collected 2, 4 and 24 hours after the administration. The collected urine was filtered through a filter having a pore size of $0.45 \mathrm{~mm}$ (Millipore) and was mixed with an equivalent volume of $50 \% \mathrm{CH}_{3} \mathrm{CN}-0.05 \mathrm{M}$ phosphate buffer ( $\mathrm{pH}$ 6.5) to serve as an urine sample. The bioassay was carried out by $M$. luteus to determine the concentration of the test compound in the urine sample and the recovery in the urine was calculated. 